\title{
Dairy Product Intake Modifies MicroRNA Expression among Individuals with Hyperinsulinemia: A Post-Intervention Cross-Sectional Study
}

\author{
Leila Khorraminezhad $^{a}$ Iwona Rudkowska ${ }^{b}$ \\ aEndocrinology and Nephrology Unit, CHU de Québec-Laval University Research Center, Quebec, QC, Canada;

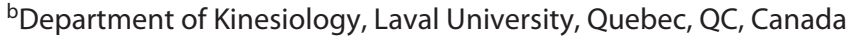

\section{Keywords}

Dairy product $\cdot$ microRNA $\cdot$ Hyperinsulinemia $\cdot$ Type 2

diabetes

\begin{abstract}
Introduction: MicroRNA (miRNA) profiles have been shown to change after intake of dairy products. Dysregulation of miRNA is associated with the changes in the level of glycemic parameters. The objectives are: (1) to investigate miRNA expression after consumption of dairy products and (2) to study the association between miRNAs and glycemic profile among individuals with hyperinsulinemia. Methods: In crossover design, 24 participants were randomized into 2 phases: high dairy (HD) ( $\geq 4$ servings/day according to the Canadian food guide [2007]) and adequate dairy (AD) ( $\leq 2$ servings/day) over 6 weeks. First, miRNAs were extracted from a pooled plasma sample of 10 subjects after HD and AD intervention which analyzed in duplicate by array hybridization (Affymetrix Gene Chip miRNA Array v. 4.0). Second, 6 miRNAs related to type 2 diabetes (T2D) were validated by quantitative reverse transcription polymerase chain reaction (qRT-PCR) from plasma of 24 participants. Results: Microarray analysis indicated that 297 miRNAs expressed differentially ( $F C \geq \pm 1.2$; $p$ value $<0.05$ ) in a pooled plasma sample of 10 subjects. Among pooled miRNAs, the level of selected miRNAs, including miR-652-3p, miR-106b-5p, miR-
\end{abstract}

93-5p, and miR-107 were downregulated; conversely, miR223-3p and miR-122-5p were upregulated. After qRT-PCR validation, only the expression level of miR-106-5p tended to be increased after HD compared to AD $(p=0.06)$. After AD intervention, the level of fasting plasma glucose (FPG) and insulin and homeostatic model assessment of insulin resistance were negatively correlated with miR-122-5p. Similarly, negative correlation was found between miR-106-5p and FPG. Conclusion: The miRNAs profile was modified after HD compared to $A D$, and this may have role in modifying the risk of T2D (registration No. NCT02961179).

(C) 2022 The Author(s) Published by S. Karger AG, Basel

\section{Introduction}

Hyperinsulinemia is a common disorder among individuals with obesity and metabolic syndrome [1]. Furthermore, hyperinsulinemia is defined as an elevated circulating insulin along with dysfunction of beta-cells causes a deficiency of insulin secretion and type 2 diabetes (T2D) [2]. The global prevalence of T2D is estimated to increase from $9.3 \%$ (463 million people) in 2019 to $10.9 \%$ (700 million) by 2045 [3]; therefore, the prevention of T2D is considered as an important global health concern. In addition, genetic background and dietary intake are involved in T2D pathophysiology.
C 2022 The Author(s).

Published by S. Karger AG, Basel

This article is licensed under the Creative Commons Attribution 4.0 International License (CC BY) (http://www.karger.com/Services/ OpenAccessLicense). Usage, derivative works and distribution are permitted provided that proper credit is given to the author and the original publisher.
Correspondence to:

Iwona Rudkowska, iwona.rudkowska@crchudequebec.ulaval.ca 
The microRNAs (miRNAs) are one of the major epigenetic regulators of gene expression. miRNAs are 19-23 nucleotides, non-coding, and endogenous RNAs [4] which are expressed in different tissues such as adipose tissue, the liver, the skeletal muscle, the heart, and blood [5], and circulated in the plasma, serum, and tissues [6]. The miRNAs play relevant epigenetic roles to control the expression level of genes. Changes in expression of several miRNAs have been associated with higher plasma glucose level and lower insulin resistance (IR) in subjects with T2D [4, 7]. For example, upregulation of miR-223 in beta-cells positively correlated to improve beta-cell proliferation and insulin secretion among diabetic and prediabetic individuals $[8,9]$. Moreover, a meta-analysis of case-control studies demonstrated that serum levels of miR-652 [10] and miR-93 [11] were downregulated among patients with T2D, while an increase in serum level of miR-122 was associated with the developing IR, metabolic syndrome, and T2D [12]. Thus, incidence of T2D may be affected by regulation of miRNAs.

Regulation of miRNAs and many physiological processes are strongly affected by dietary intake. It remains unclear that how intake of dairy products and its nutrients modulated the miRNAs regulation. Recent cohort study of healthy adults indicated that intake of vitamin D as main nutrient in dairy products was negatively associated with the expression levels of miR-1277 and miR-144$3 p$ [13]. Further, after supplementation by vitamin $D$, the expression level of 136 miRNAs was changed and a positive correlation was found between the serum level of vitamin D and miR-532-3p and miR-342-5p [14]. Additionally, a randomized crossover study demonstrated that after intake of different quantities $(0.25,0.5,1 \mathrm{~L})$ of milk, the expression level of miR-29b was upregulated [15]. The ability of dairy products and its nutrients to regulate serum miRNAs was indicated in the previous studies.

Despite the effect of dairy intake on hyperinsulinemia and miRNA expression separately, the association between miRNA expression and glycemic profile after dairy intake is still unknown. This study investigates the changes in the expression of miRNAs after consumption of dairy products. Additionally, the association between expression of miRNAs and glycemic profile among individuals with hyperinsulinemia is examined.

\section{Methods and Materials}

A total of 24 overweight $(25<$ body mass index [BMI] $\leq 29.9 \mathrm{~kg} /$ $\mathrm{m}^{2}$ ) or obese (BMI $\left.\geq 30 \mathrm{~kg} / \mathrm{m}^{2}\right)$ adults ( 17 men and 7 women) with hyperinsulinemia (fasting insulin $>90 \mathrm{pmol} / \mathrm{L}$, fasting glucose $<7.0$ $\mathrm{mmol} / \mathrm{L}$, glycated hemoglobin $<6.5 \%)$ were recruited for this study at the Centre Hospitalier Universitaire (CHU) de Québec - Université Laval Research Center in Québec City, Canada, via poster advertisements, flyers, and email lists from Université Laval and from the Institute of Nutrition and Functional Foods. To provide $80 \%$ power to find an anticipated difference ( $11 \%)$ in insulin sensitivity, the minimum size $(n=24)$ was calculated to provide $80 \%$ power [16]. Exclusion criteria were consumption of high dairy (HD) at baseline (>2 servings/day), intolerance or allergy to dairy products, those who had a diagnosis of $\mathrm{T} 2 \mathrm{D}$, major surgery in 3 months before the study, inflammatory bowel disease, gastrointestinal disorders, thyroid disorders, intake of any medication which have effect on glucose metabolism, blood pressure, and lipid profile. All participants were educated to perform in the same protocols for diet and exercise during a 3-month period before the onset of the study. The diagram of the study was described in online supplementary Figure 1 (for all online suppl. material, see www. karger.com/doi/10.1159/000523809). Further, the Consolidated Standards of Reporting Trials statement was completed to improve the reporting of the present study (online suppl. Table 1).

\section{Dietary Intervention}

In this study, participants were equally randomized either to $\mathrm{HD}$ ( $\geq 4$ servings/day, according to the serving sizes recommended by Canada's Food Guide for Healthy Eating [2007]) or adequate dairy (AD) ( $\leq 2$ servings/day) for 6 weeks, separated by a 6 -week washout period. Consumption of dairy products included milk, yogurt, cheese, kefir, cream ( $\leq 15 \%$ fat content) [17], and ice cream (1 serving equals $125 \mathrm{~mL}$ ) was limited to 3 servings/week. Butter, milk substitutes and derivatives, and whipped cream or cream $>15 \%$ fat content were not accepted as dairy products in the daily serving count due to higher percentage of total fat \%. All participants were asked to keep their usual dietary habits and physical activity. Examples of dairy products and serving sizes were suggested using the recommendations of Canada's Food Guide for Healthy Eating 2007. Dietary intake was collected at each visit by using a validated food frequency questionnaire containing 91 items and 33 sub-questions that were completed through a webplatform linked to the nutrition data system for research [16]. The dietary intake was estimated using the Canadian nutrient file 2015 [18].

\section{Anthropometric Measurements}

Weight was measured with the least clothes and not wearing shoes with the use of a digital weighing scale with $100 \mathrm{~g}$ accuracy (Health O Meter Professional; Sunbeam Products, Inc., Boca Raton, FL, USA). Height was measured with $1 \mathrm{~mm}$ accuracy using a stadiometer (the Easy-Glide Bearing Stadiometer; Perspective Enterprises, Portage, MI, USA). BMI was calculated as weight $(\mathrm{kg})$ divided by height $(\mathrm{m})$ squared and recorded as $\mathrm{kg} / \mathrm{m}^{2}$.

\section{Clinical Measurements}

Four visits were scheduled at the beginning and at the end of each intervention period. Serum fasting glucose and insulin levels were measured using hexokinase assays [19] and chemiluminescence immunoassays [20], respectively. Homeostatic model assessment of IR (HOMA-IR) was calculated according to a standard formula: $=($ insulin $[\mathrm{pmol} / \mathrm{L}] \times$ glucose $[\mathrm{mmol} / \mathrm{L}]) / 135$ [21]. Plasma total cholesterol and triglycerides (TG) concentrations were measured using enzymatic assays $[22,23]$. The HDL cholesterol 
Table 1. Main characteristic at the end of each phase (AD or HD intake) among subjects with hyperinsulinemia

\begin{tabular}{|c|c|c|c|}
\hline Characteristics & $\begin{array}{l}A D \\
(\text { mean } \pm S D)\end{array}$ & $\begin{array}{l}\mathrm{HD} \\
(\text { mean } \pm \mathrm{SD})\end{array}$ & $\begin{array}{l}p \text { value } \\
\text { (paired student's } \\
t \text { test) }\end{array}$ \\
\hline \multicolumn{4}{|c|}{ Participants in validation process (RT-PCR) $(N=24)$} \\
\hline BMI $\left(\mathrm{kg} / \mathrm{m}^{2}\right)$ & $31.05 \pm 3.23$ & $31.17 \pm 3.18$ & 0.41 \\
\hline FPG (mmol/L) & $5.29 \pm 0.61$ & $5.32 \pm 0.63$ & 0.79 \\
\hline Fasting blood insulin (pmol/L) & $125.09 \pm 75.46$ & $129.68 \pm 73.78$ & $0.76^{* *}$ \\
\hline HOMA-IR & $5.28 \pm 4.01$ & $5.18 \pm 3.51$ & $0.90 * *$ \\
\hline $\mathrm{HDL}(\mathrm{mmol} / \mathrm{L})$ & $1.07 \pm 0.25$ & $1.09 \pm 0.26$ & 0.62 \\
\hline $\mathrm{LDL}(\mathrm{mmol} / \mathrm{L})$ & $2.42 \pm 0.94$ & $2.57 \pm 1.00$ & 0.28 \\
\hline $\mathrm{TG}(\mathrm{mmol} / \mathrm{L})$ & $1.70 \pm 1.16$ & $1.68 \pm 0.96$ & $0.79^{* *}$ \\
\hline $\mathrm{TC}(\mathrm{mmol} / \mathrm{L})$ & $4.28 \pm 1.04$ & $4.44 \pm 1.19$ & 0.32 \\
\hline \multicolumn{4}{|c|}{ Participants in screening process (microarray analysis) $(N=10)$} \\
\hline BMI $\left(\mathrm{kg} / \mathrm{m}^{2}\right)$ & $31.06 \pm 2.45$ & $31.20 \pm 2.58$ & 0.39 \\
\hline $\mathrm{FPG}(\mathrm{mmol} / \mathrm{L})$ & $5.25 \pm 0.36$ & $5.37 \pm 0.45$ & 0.54 \\
\hline Fasting blood insulin (pmol/L) & $121.77 \pm 68.50$ & $121.44 \pm 63.90$ & 0.97 \\
\hline HOMA-IR & $4.74 \pm 2.57$ & $4.84 \pm 2.43$ & 0.79 \\
\hline $\mathrm{HDL}(\mathrm{mmol} / \mathrm{L})$ & $1.12 \pm 0.19$ & $1.11 \pm 0.23$ & 0.76 \\
\hline $\mathrm{LDL}(\mathrm{mmol} / \mathrm{L})$ & $2.66 \pm 1.07$ & $2.65 \pm 1.33$ & 0.95 \\
\hline $\mathrm{TG}(\mathrm{mmol} / \mathrm{L})$ & $1.55 \pm 0.52$ & $1.53 \pm 0.42$ & 0.87 \\
\hline $\mathrm{TC}(\mathrm{mmol} / \mathrm{L})$ & $4.50 \pm 1.20$ & $4.46 \pm 1.54$ & 0.89 \\
\hline
\end{tabular}

Values are means $\pm S D$ s, paired $t$ test. $B M I$, body mass index; FPG, fasting plasma glucose; HOMA-IR, homeostatic model assessment of insulin resistance; HDL, high-density lipoproteins; LDL, low-density lipoproteins; TG, triglycerides; TC, total cholesterol. * $p<0.05$ significant. ${ }^{* *}$ Wilcoxon test (nonparametric variable). fraction was obtained after precipitation of very low-density lipoprotein and LDL particles in the infranatant with heparin manganese chloride [24]. LDL-C was calculated with the Friedewald formula [17].

Screening Phase: miRNA Extraction and Microarray Analysis

Plasma of 10 randomly selected subjects for each intervention (AD and $\mathrm{HD}$ ) was pooled in two samples (each $2 \mu \mathrm{L}$ ). miRNAs in these 2 samples were isolated using the NucleoSpin ${ }^{\circledR}$ miRNA plasma extraction kit (Micherey-Nagel). After, quantification by Bioanalyzer (Agilent) each pooled sample (AD and HD) contained approximately $280 \mathrm{ng}$ of total RNA. Therefore, $140 \mathrm{ng}$ of RNA was analyzed for each microarray chip (Affymetrix GeneChip miRNA Array v. 4.0) according to manufacturing instructions at the $\mathrm{CHU}$ de Quebec Research Center. The samples were analyzed in duplicate.

Validation Phase: MiRNA Extraction and Quantitative

Reverse-Transfer Real-Time PCR Analysis

The miRNAs were isolated from $300 \mu \mathrm{L}$ of plasma of each 24 subjects using the NucleoSpin ${ }^{\circledR}$ miRNA plasma extraction kit (Micherey-Nagel). The miRNAs extraction was done following the manufacturer recommendations and the concentrations were determined by NanoDrop (Thermo Scientific, Waltham, MA, USA) (between 7 and $20 \mathrm{ng} / \mu \mathrm{L}$ ). The cDNA preparation was performed using the TaqMan ${ }^{\circledR}$ Advanced miRNA cDNA Synthesis Kit (applied biosystems, Waltham, MA, USA). The TaqMan ${ }^{\circledR}$ Advanced miRNA Assays with primers detecting: miR-223-3p, miR-652-3p, miR-93-5p, miR-106b-5p, miR-122-5p, and miR-107 (applied bio- systems) were used to analyze miRNA expression levels using realtime PCR instrument (applied biosystems). Equal amounts of miR-191-5p were added to each sample as an exogenous control for assessment of isolation efficiency, as well as for normalization of expression levels of analyzed miRNAs. Samples were analyzed in triplicates. The relative expression was calculated using the following formula: $\Delta \mathrm{CT}=$ average $\mathrm{CT}_{\text {test miRNA }}-$ average $\mathrm{CT}_{\text {internal control }}$ and CT stands for cycle threshold.

\section{Statistical Analysis}

Statistical analyses were performed using IBM SPSS statistics software for macOS version 27.0.1.0 (IBM Corp., Armonk, NY, USA). Before conducting statistical analyses, the Shapiro-Wilk test was examined to evaluate the normality of the data distribution. Comparison of baseline parameters (anthropometric parameters, lipid profile [LDL, HDL, TG, Chol]), glycemic profile (fasting plasma glucose [FPG], fasting insulin, and HOMA-IR) at the beginning of each phase of the intervention (AD or HD) was conducted using a paired $t$ test and Wilcoxon signed rank for parametric and nonparametric variables, respectively. The Pearson and Spearman bivariate correlation was conducted between levels of miRNAs and glycemic parameters. Partial correlation adjusted for age, sex, and BMI were performed to examine the quantitative relationship between the expression levels of miRNAs and glycemic profiles. Furthermore, correlation analysis was used to examine the association between expression levels of miRNAs and intake of micronutrient in each phase (after $\mathrm{AD}$ and $\mathrm{HD}$ ). The heatmap and charts were generated by GraphPad Prism version 9 for macOS. $p<0.05$ was considered to indicate a statistically significant difference. 
Table 2. Dietary intake after AD product intake and HD product intake in subjects with hyperinsulinemia

\begin{tabular}{|c|c|c|c|}
\hline Dietary intake & $A D$ & HD & $p$ value \\
\hline \multicolumn{4}{|c|}{ Participants in validation process (RT-PCR) $(N=24)$} \\
\hline Total energy intake (kcal/day) & $2,110.94 \pm 792.70$ & $2,473.37 \pm 929.65$ & $0.003^{*}$ \\
\hline Total carbohydrate intake (g/day) & $239.14 \pm 100.47$ & $279.39 \pm 114.31$ & $0.008 *$ \\
\hline Total protein intake (g/day) & $90.96 \pm 31.71$ & $116.73 \pm 36.84$ & $<0.001^{*}$ \\
\hline Total fat intake (g/day) & $83.95 \pm 34.07$ & $95.51 \pm 40.85$ & 0.064 \\
\hline \multicolumn{4}{|l|}{ Participants in screening process $(N=10)$} \\
\hline Total energy intake (kcal/day) & $2,160.49 \pm 765.30$ & $2,741.07 \pm 1,051.83$ & $0.006^{*}$ \\
\hline Total carbohydrate intake (g/day) & $240.58 \pm 86.06$ & $302.05 \pm 137.07$ & $0.026^{*}$ \\
\hline Total protein intake (g/day) & $89.34 \pm 26.76$ & $128.79 \pm 33.38$ & $<0.001^{*}$ \\
\hline Total fat intake (g/day) & $84.80 \pm 33.90$ & $107.51 \pm 47.21$ & $<0.001^{*}$ \\
\hline
\end{tabular}

Means $\pm S D$, Dietary intake was extracted from food frequency questionnaire. FFQ, food frequency questionnaire. Paired sample $t$ test, $* p<0.05$ significant.

\section{Results}

\section{Descriptive Characteristics between $A D$ and $H D$}

Twenty-four overweight or obese adults with hyperinsulinemia were randomly selected to analyse in this crossover study (age $56.2 \pm 13.7$ years, BMI $31.21 \pm 2.53 \mathrm{~kg} /$ $\mathrm{m}^{2}$ ). Ten subjects were selected for screening process (age $54.1 \pm 14.5$ years, BMI $31.81 \pm 1.83 \mathrm{~kg} / \mathrm{m}^{2}$ ). Table 1 presented an anthropometric and clinical parameters of study participants following each of two interventions ( $\mathrm{HD}, \geq 4$ servings/day; $\mathrm{AD}$, ' $\leq 2$ servings/day) in both microarray process and qRT-PCR screening process. The participants' flow diagram was presented in the previously published study [16] (online suppl. Fig. S1). No baseline differences were demonstrated in lipid profile (LDL, HDL, TG, Chol), glycemic profile (fasting insulin, FPG, HOMA-IR), and anthropometric criteria at the beginning of each intervention (AD and HD) $(p>0.05)$ among all participants in validation and screening process.

\section{Comparison of Dietary Intake between $A D$ and $H D$}

Dietary intake after AD and HD were presented for all participants in validation $(n=24)$ and selected participants in screening $(n=10)$ phases in Table 2. Mean of dairy consumption after $\mathrm{HD}$ and $\mathrm{AD}$ were $6.0 \pm 0.6$ and $1.6 \pm 1.4$ per day, respectively among 24 subjects and 5.4 \pm 0.8 and $1.4 \pm 0.9$ servings for 10 participants in screening phase. Total intake of energy, carbohydrate, protein, and fat were increased significantly after HD compared to $\mathrm{AD}(p>0.05)$ in participants in validation process. Similar results were found for the selected participants in the screening process.

\section{MiRNA Screening}

Microarray analysis indicated that 297 miRNAs were differentially expressed between $\mathrm{AD}$ and $\mathrm{HD}$ (137 under expressed $(\mathrm{FC} \leq-1.2)$ and 160 overexpressed $(\mathrm{FC} \geq 1.2)$. Top downregulated miRNAs were included miR-652-3p $(\mathrm{FC}=-3.34, p=0.001), \mathrm{miR}-106 \mathrm{~b}-5 \mathrm{p}(\mathrm{FC}=-1.37, p=$ $0.04)$, miR-93-5p $(\mathrm{FC}=-1.88, p=0.006), \mathrm{miR}-107(\mathrm{FC}=$ $-1.39, p=0.01)$ and upregulated-miRNAs such as miR$223-3 \mathrm{p}(\mathrm{FC}=4.31, p=0.01)$, and $\mathrm{miR}-122-5 \mathrm{p}(\mathrm{FC}=1.33$, $p=0.02)$. The association of these top up- and downregulated $(\mathrm{FC} \geq 1.2 \mathrm{FC} \leq-1.2)$ miRNAs with $\mathrm{T} 2 \mathrm{D}$ were examined in previous studies, which are summarized in Table 3 .

Furthermore, pathway analysis was done using http:// mirdb.org. The heatmap indicated that miR-106-5p plays roles in many metabolic pathways, especially TGF-beta signaling pathway, MAPK signaling pathway, endocytosis, and cancer pathways. Most of the identified pathways are associated with the inflammation as a major consequent of T2D (Fig. 1).

\section{Comparison in the Plasma Level of miRNAs between $A D$ and $H D$ Interventions}

The qRT-PCR validation was performed to quantify the differential expressed level of the six miRNAs. Further, the results of qRT-PCR were normalized by housekeeping miR-191-5p (FC=0.01, $p=0.93)$. The miR-191$5 p$ was considered as housekeeping miRNA due to high expression level, low standard deviation in both intervention $(\mathrm{AD}=6.93 \pm 0.06$ and $\mathrm{HD}=6.93 \pm 0.20)$, FC close to 1 or $1(\mathrm{FC}=1.01)$, and $p$ value close to $1(p=0.93)$. The expression level of miR-106-5P tended to be higher $(p=$ 0.06 ) after $\mathrm{HD}$ intake compared to AD. No significant dif-
Khorraminezhad/Rudkowska 


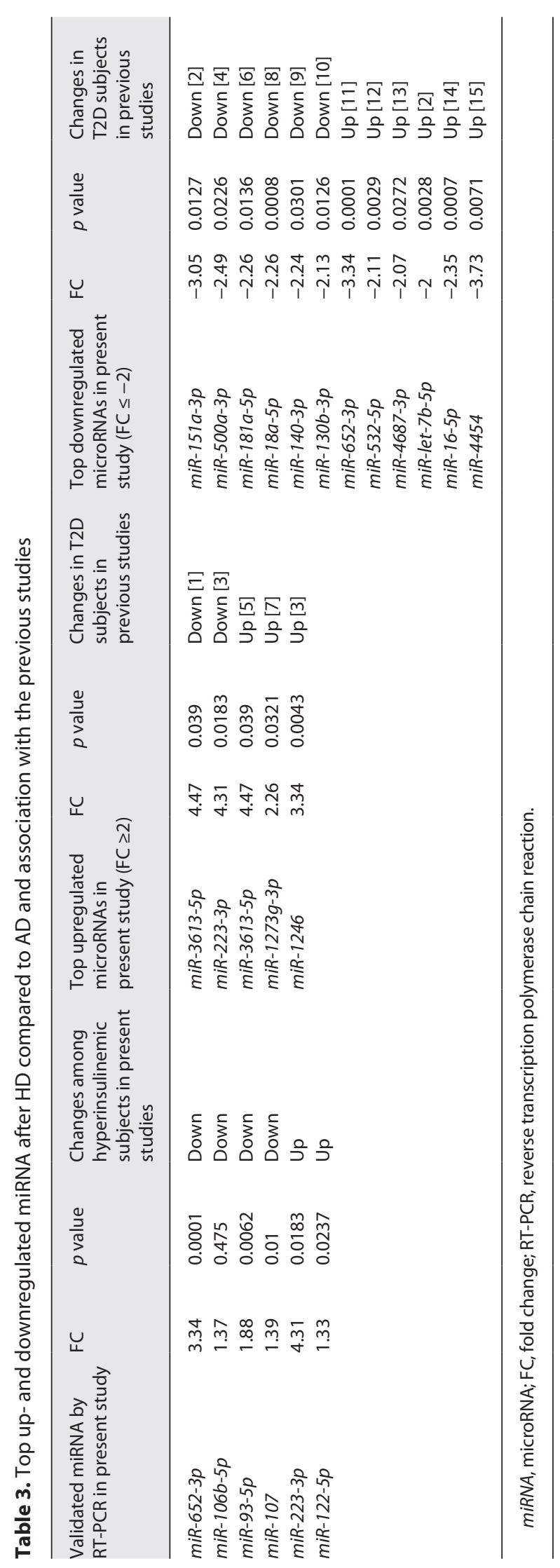

Effect of Dairy Intake on MicroRNA Expression ferences were found in the expression levels of miR-2233p, miR-652-3p, miR-93-5p, miR-107, and miR-122.5p $(p>0.05)$ (Fig. 2).

\section{Correlation between miRNA and Glycemic \\ Parameters after HD Compared to AD}

Correlation analysis was conducted as a post-intervention cross-sectional investigation. Bivariate correlation indicated a negative correlation between expression level of miR-122-5p and FPG ( $p=0.018, r=-0.490)$, fasting insulin $(p=0.016, r=-0.517)$, and HOMA-IR $(p=0.003$, $r=-0.588)$ as well as between miR-106-5p and FPG ( $p=$ $0.022, r=-0.466)$. After HD consumption, toward negative correlation was demonstrated between miR-652-3p $(p=0.085, r=-0.375)$ and miR-93-5p ( $p=0.077, r=$ $-0.385)$ with fasting insulin. By adjusting for age, sex, and $\mathrm{BMI}$, negative correlation between miR-122-5p and FPG $(p=0.007, r=-0.641)$, fasting insulin $(p=0.007, r=$ $-0.645)$, and HOMA-IR ( $p=0.003, r=-0.698)$ remained significant after $A D$. Furthermore, negative correlation between miR-106-5p with FPG ( $p=0.02, r=-0.576)$ was demonstrated after AD. Moreover, after HD intervention, negative correlation was observed between miR223-3p with HOMA-IR ( $p=0.02, r=-0.551)$ and between miR-93-5p and fasting blood insulin $(p=0.03, r=$ $-0.536)$ (Fig. 3).

\section{Discussion}

Overall, 297 miRNAs were differentially expressed in a pooled plasma sample of $\mathrm{AD}$ and $\mathrm{HD}$ intervention. Top downregulated miRNAs were included miR-652-3p, miR-106b-5p, miR-107, and miR-93-5p, together with upregulation of miR-223-3p and miR-122-5p. Moreover, after validation phase, the expression level of miR-106-5P was increased after $\mathrm{HD}$ compared to $\mathrm{AD}$. After $\mathrm{AD}$, the correlation analysis indicated that the expression level of miR-122-5P were negatively associated to FPG, fasting insulin, and HOMA-IR. Moreover, After AD, a negative correlation between miR-106-5p with FPG was indicated, similar results were found between miR-223-3p and miR93-5p with HOMA-IR and fasting blood insulin, respectively.

The microarray analysis indicated that the regulation of number of 160 and 137 miRNAs was changed; in particular, the expression level of miR-652-3p, miR-106b-5p, and miR-93-5p, miR-107 were downregulated, while miR-223-3p and miR-122-5p were upregulated in the pooled samples analysis. Following studies indicated that 


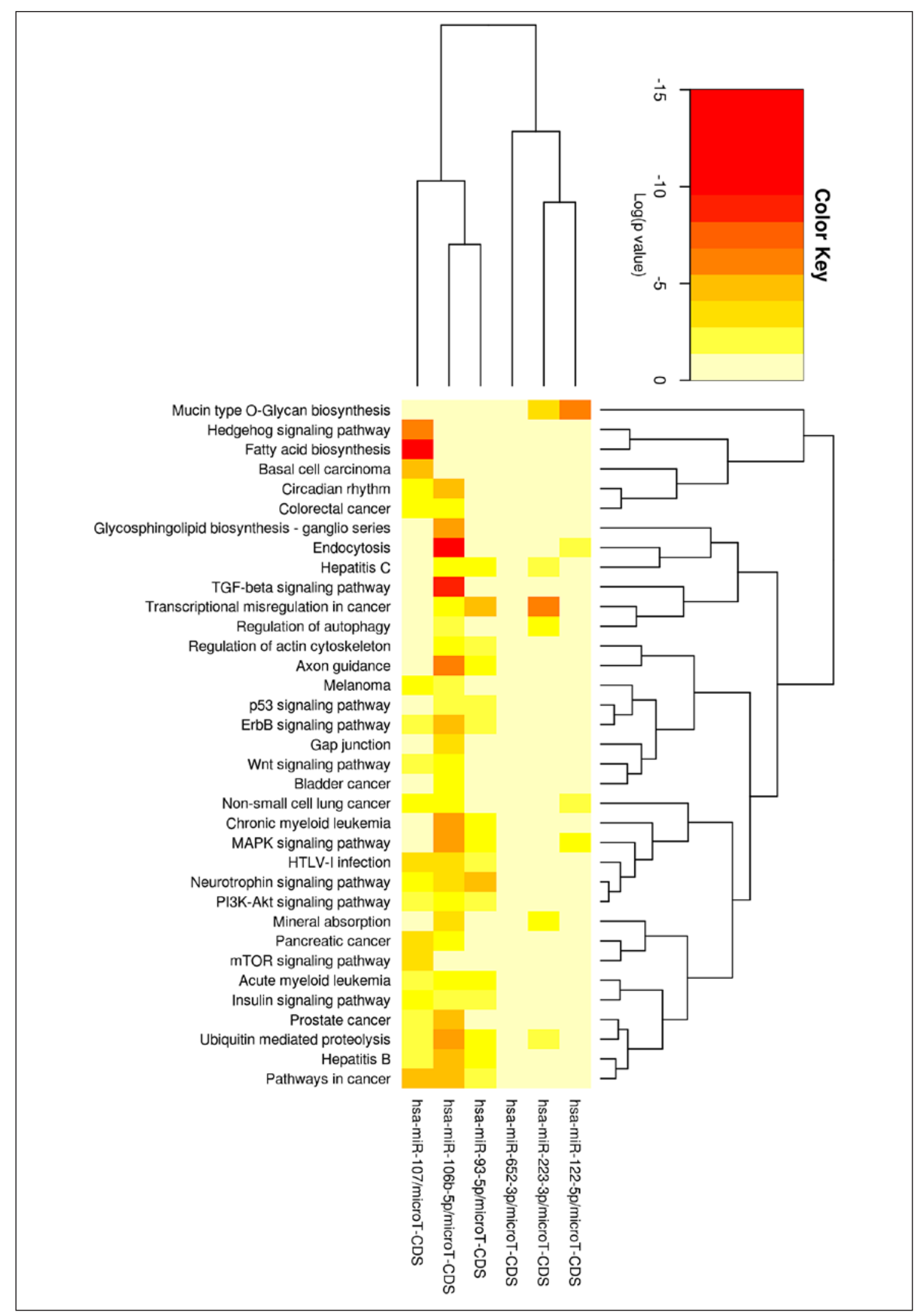

Fig. 1. Pathways associated to inflammation as a major consequent of T2D. 
Fig. 2. Plasma levels of miRNAs after HD and $\mathrm{AD}$ (paired $t$ test and Wilcoxon). ${ }^{*} p<$ 0.05 .

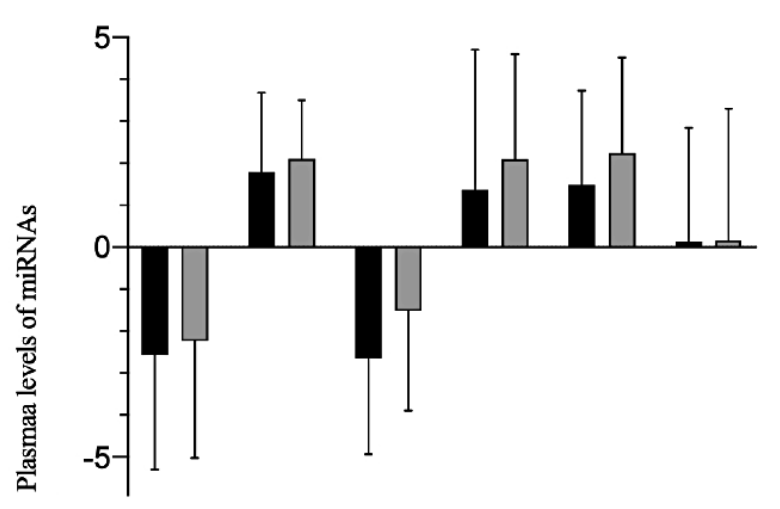

Adequate-Dairy

$\square$ High-Dairy

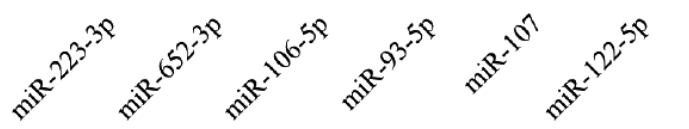

miRNA validated by qRT-PCR
Fig. 3. Association between plasma level of miRNAs and glycemic parameters after consumption of AD and HD. Partial correlation after adjusted for age, sex, and BMI, ${ }^{*} p<0.05$ significant.

Effect of Dairy Intake on MicroRNA Expression
Lifestyle Genomics 2022;15:77-86

High-dairy

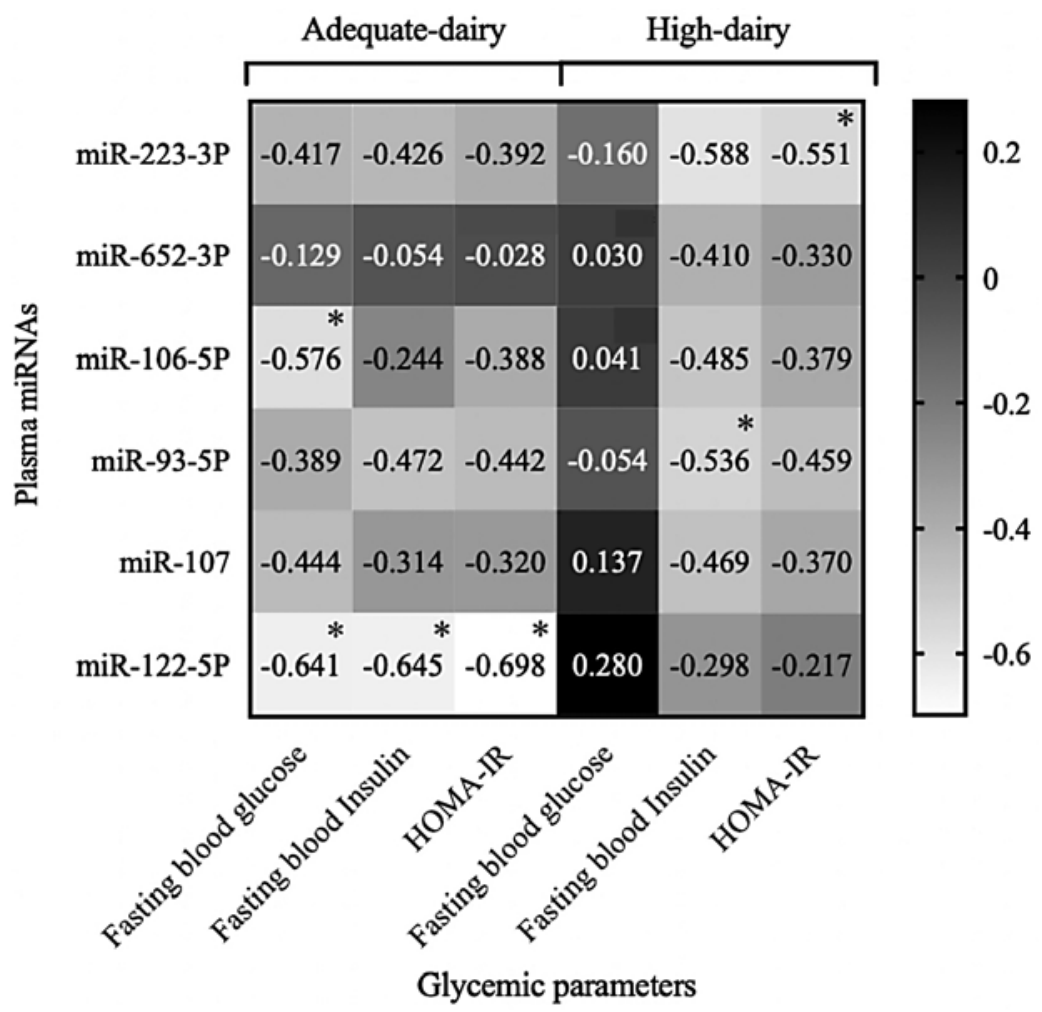


regulation of miRNAs can change by dairy intake and its nutrient, including calcium, vitamin $\mathrm{D}$, whey and casein protein. Inconsistent with the present study, a validation study on the plasma of healthy adults demonstrated no evidence of the effect of milk on 223 miRNAs examined after milk ingestion [18]. Microarray analysis of plasma sample of adults who consumed high dose of vitamin $\mathrm{D}$ (20,000-40,000 IU/w - 12 months) demonstrated that 136 miRNAs were expressed differentially and positive correlation was found between vitamin D and miR-532$3 p$ [14]. In sum, dairy intake and its associated nutrients can alter the miRNAs profile.

After validation miRNAs by qRT-PCR, the expression level of miR-106-5p was increased without changes in miR-223-3p, miR-122-5p, miR-93-5p, miR-107, and miR-652-3p after HD compared to AD. A study withinsubject design indicated that the plasma level of miR106b-5p and miR-223-3p expression were increased by $80 \%$ after consumption of 1-4 servings/day milk compared to baseline control in healthy individuals [25]. Similarly, an experimental study demonstrated that after intake of diet high in ruminant trans fatty acids $(3.7 \%$ of total energy/day) such as meat and dairy products, high level of miR-223-3p in plasma HDL were positively correlated with high level of C-reactive protein among healthy men and increased the risk of inflammation and cardiovascular disorders [26]. In addition, other studies provide proof which the miRNAs might change by intake of dietary intake. For example, the expression levels of miR-146a, miR-150, and miR-155 were inhibited strongly by $1.25(\mathrm{OH}) 2 \mathrm{D}$, especially in rats which feed high-fat diet ( $45 \%$ fat of total energy) and supplemented with vitamin D (15,000 IU/kg of food for 10 weeks) [27]. Moreover, the expression levels of miR-146a and miR-558 were downregulated; however, miR-124a and miR-155 were increased among participants with vitamin $\mathrm{D}$ deficiency and severe lower back pain in a cross-sectional study [28]. Further, the regulation of miR-221 [29] and miR-9 [30] play a key checkpoint in the homeostasis of calcium and phosphate. And the high expression of miR-155 has been downregulated by intake of high doses of vitamin D [31]. More, vitamin D intake was negatively correlated with the expression of miR-1277-5p and miR-144-3p in a cohort study of healthy adults [13]. Overall, previous studies highlighted dairy nutrients and dietary intake which have impact on circulating miRNAs profile.

The correlation analysis indicated that miR-122-5p and miR-106-5p were negatively correlated with the plasma levels of FPG, fasting insulin, and HOMA-IR after AD. A similar correlation was found between miR-223-
$3 p$ with fasting insulin and HOMA-IR after HD. In contrast to current study, a 30-year follow-up cohort study demonstrated that the upregulation of serum level of miR-122-5p positively correlated with IR among T2D patients compared to healthy individuals [32]. Therefore, reduction in the expression level of miR-122-5p expression is associated with related genes in glycolysis and pyruvate kinases [33]. Furthermore, a case-control study indicated that the plasma level of miR-122-5p was downregulated among obese diabetic individuals after Roux-en-Y gastric bypass surgery, while upregulated among diabetic patients with obesity $(B M I>30)$ [34]. Moreover, miR-223-3p was downregulated among T2D patients and was considered as a potential circulating biomarker of T2D [10]. In addition, miR-223 regulates glucose transporter 4 (Glut4) expression and beta-cell proliferation to keep normal glucose uptake [35] and promote beta-cell proliferation [36]. The increasing the miR-223-3p after HD could be associated to decrease the risk of T2D development. Moreover, plasma level of miR$106 \mathrm{~b}$ has role in insulin metabolism and the skeletal muscle, which downregulated among adults with T2D compared to non-T2D, over 10-year follow-up cohort study [37]. Furthermore, miR-106b plays role in promote betacell regeneration and reduces hyperglycemia [38]. The upregulation of miR-106b was associated to the reduction of pro-inflammatory cytokines such as IL- $1 \beta$, IL- 6 , and TNF- $\alpha$ [39]. Therefore, longer intervention times might be needed to have effect of dairy consumption on glucose subjects.

Overall, this study has limitations. First, the use of pooling in microarray analysis is considered a limitation because of the inability to identify and appropriately transform or remove aberrant subjects and the inability to estimate within population variation [40]. Second, the measurement of miRNA levels at the beginning of each phase was not included. Third, only selected miRNAs were validated in current study which more studies need to be done to examine additional miRNAs associated to T2D. Finally, longer studies should be done to examine the effects of longer intervention with different types of dairy products on the expression level of miRNAs be enough sufficient to modify the glycemic profile and reduce the risk of T2D and hyperinsulinemia because studies less than 8 week of dairy product intervention indicated no significant changes in insulin sensitivity, whereas studies between 12-week and 24-weeks resulted in a favorable effect of higher dairy intake on insulin sensitivity [41]. 
In conclusion, the results indicate that the expression levels miRNAs changed after intake of 4 servings/day or more dairy products compared to 2 servings/day or less, to potentially decrease the risk of development hyperinsulinemia through the modification of pathways and expression level of miRNAs associated to T2D.

\section{Acknowledgments}

We thank Sarah O'Connor, Andréa Taschereau-Charron, Sarah Chouinard-Castonguay, Élise Cant, Valérie-Ėve Julien, and Camille Lambert, for their help with the visits with participants. We also thank Abderrahim Benmoussa, Imane-Rockya Chaouch, Sara Michel, and Catherine Raymond for their knowledge and help with the laboratory work.

\section{Statement of Ethics}

The study has been approved by the CHU de Québec-Université Laval Research Center Ethics Committees (permission code: 2017-3228) and conducted in accordance with the ethical guidelines of the Declaration of Helsinki. Written informed consent was obtained from all individuals prior to the start of the study. The information was collected between February 2017 to July 2018. This trial protocol is registered in Clinical Trial.gov: NCT02961179.

\section{Conflict of Interest Statement}

Iwona Rudkowska is an associate editor of "Lifestyle Genomics." However, other authors declare no conflict of interest.

\section{Funding Sources}

This study was funded by the Canadian Institutes of Health Research (CIHR Grant No. PJT-364008 to I.R.). L.K. received a scholarship from CHU de Québec-Laval University Research Center and Fonds de recherche du Québec - Nature et les technologies; I.R. hold a Junior 2 research Scholar from the Fonds de recherche du Québec - Santé (FRQ-S).

\section{Author Contributions}

I.R. was involved in the conceptualization of the study and resources; I.R. and L.K. were responsible for methodology and data analyses and writing the original draft; I.R. was involved in the reviewing and editing the manuscript and responsible for supervision and funding acquisition.

\section{Data Availability Statement}

Data cannot be shared for confidentiality reasons. Queries about the data should be directed to the corresponding author.

\section{References}

1 Thomas DD, Corkey BE, Istfan NW, Apovian CM. Hyperinsulinemia: an early indicator of metabolic dysfunction. J Endocr Soc. 2019;3: 1727-47.

2 Harreiter J, Roden M. Diabetes mellitus-definition, classifikation, diagnose, screening und prävention (update 2019). Wien Klin Wochenschr. 2019;131:6-15.

3 Saeedi P, Petersohn I, Salpea P, Malanda B, Karuranga S, Unwin N, et al. Global and regional diabetes prevalence estimates for 2019 and projections for 2030 and 2045: results from the International Diabetes Federation Diabetes Atlas. Diabetes Res Clin Pract. 2019; 157:107843.

4 Hammond SM. An overview of microRNAs. Adv Drug Deliv Rev. 2015;87:3-14.

5 Feng J, Xing W, Xie L. Regulatory roles of microRNAs in diabetes. Int J Mol Sci. 2016;17: 1729.

6 O’Brien J, Hayder H, Zayed Y, Peng C. Overview of microRNA biogenesis, mechanisms of actions, and circulation. Front Endocrinol. 2018;9:402.

7 Benmoussa A, Provost P. Milk microRNAs in health and disease. Compr Rev Food Sci Food Saf. 2019;18(3):703-22.
8 Li Y, Deng S, Peng J, Wang X, Essandoh K, $\mathrm{Mu}$ X, et al. MicroRNA-223 is essential for maintaining functional $\beta$-cell mass during diabetes through inhibiting both FOXO1 and SOX6 pathways. J Biol Chem. 2019;294: 10438-48.

9 Lopez YON, Garufi G, Seyhan AA. Altered levels of circulating cytokines and microRNAs in lean and obese individuals with prediabetes and type 2 diabetes. Mol Biosyst. 2017;13:106-21.

10 Zhu H, Leung SW. Identification of microRNA biomarkers in type 2 diabetes: a metaanalysis of controlled profiling studies. Diabetologia. 2015;58:900-11.

11 Akhbari M, Shahrabi-Farahani M, Biglari A, Khalili M, Bandarian F. Expression level of circulating miR-93 in serum of patients with diabetic nephropathy. Turk J Endocrinol Metab. 2018;22:78.

12 Willeit P, Skroblin P, Moschen AR, Yin X, Kaudewitz D, Zampetaki A, et al. Circulating microRNA-122 is associated with the risk of new-onset metabolic syndrome and type 2 diabetes. Diabetes. 2017;66:347-57.
13 Ferrero G, Carpi S, Polini B, Pardini B, Nieri $\mathrm{P}$, Impeduglia A, et al. Intake of natural compounds and circulating microRNA expression levels: their relationship investigated in healthy subjects with different dietary habits. Front Pharmacol. 2020:11.

14 Jorde R, Svartberg J, Joakimsen RM, Coucheron DH. Plasma profile of microRNA after supplementation with high doses of vitamin D3 for 12 months. BMC Res Notes. 2012;5:245-8.

15 Baier SR, Nguyen C, Xie F, Wood JR, Zempleni J. MicroRNAs are absorbed in biologically meaningful amounts from nutritionally relevant doses of cow milk and affect gene expression in peripheral blood mononuclear cells, HEK-293 kidney cell cultures, and mouse livers. J Nutr. 2014;144:1495-500.

16 O'Connor S, Julien P, Weisnagel SJ, Gagnon C, Rudkowska I. Impact of a high intake of dairy product on insulin sensitivity in hyperinsulinemic adults: a crossover randomized controlled trial. Curr Dev Nutr. 2019;3: nzz083.

17 Friedewald WT, Levy RI, Fredrickson DS. Estimation of the concentration of low-density lipoprotein cholesterol in plasma, without use of the preparative ultracentrifuge. Clin Chem. 1972;18:499-502. 
18 Auerbach A, Vyas G, Li A, Halushka M, Witwer K. Uptake of dietary milk miRNAs by adult humans: a validation study. F1000Res. 2016;5:721.

19 Passey RB, Gillum RL, Fuller JB, Urry FM, Giles ML. Evaluation and comparison of 10 glucose methods and the reference method recommended in the proposed product class standard (1974). Clin Chem. 1977;23:131-9.

20 Kirk RE, Othmer Donald F, Standen A, Scott JD. Encyclopedia of chemical technology. New York: The Interscience Encyclopedia, Inc.; 1947.

21 Matthews DR, Hosker J, Rudenski A, Naylor B, Treacher D, Turner R. Homeostasis model assessment: insulin resistance and $\beta$-cell function from fasting plasma glucose and insulin concentrations in man. diabetologia. 1985;28: 412-9.

22 McNamara JR, Schaefer EJ. Automated enzymatic standardized lipid analyses for plasma and lipoprotein fractions. Clin Chim Acta. 1987; 166:1-8.

23 Burstein M, Samaille J. On a rapid determination of the cholesterol bound to the serum alpha- and beta-lipoproteins. Clin Chim Acta. 1960;5:609.

24 Ash KO, Hentschel WM. High-density lipoproteins estimated by an enzymatic cholesterol procedure, with a centrifugal analyzer. Clin Chem. 1978;24:2180-4.

25 Mutai E, Ramer-Tait AE, Zempleni J. MicroRNAs in bovine milk exosomes are bioavailable in humans but do not elicit a robust pro-inflammatory cytokine response. ExRNA. 2020;2(1):1-9.

26 Desgagné V, Guay SP, Guérin R, Corbin F, Couture P, Lamarche B, et al. Variations in HDL-carried miR-223 and miR-135a concentrations after consumption of dietary trans fat are associated with changes in blood lipid and inflammatory markers in healthy men-an exploratory study. Epigenetics. 2016;11:438-48.

27 Karkeni E, Bonnet L, Marcotorchino J, Tourniaire F, Astier J, Ye J, et al. Vitamin D limits inflammation-linked microRNA expression in adipocytes in vitro and in vivo: a new mechanism for the regulation of inflammation by vitamin D. Epigenetics. 2018;13:15662.

28 Al-Rawaf HA, Gabr SA, Alghadir AH. Vitamin $\mathrm{D}$ deficiency and molecular changes in circulating microRNAs in older adults with lower back pain. Pain Res Manag. 2021;2021: 6662651.

29 Mackenzie NC, Staines KA, Zhu D, Genever P, Macrae VE. miRNA-221 and miRNA-222 synergistically function to promote vascular calcification. Cell Biochem Funct. 2014;32: 209-16.

30 Clement T, Salone V, Charpentier B, Jouzeau JY, Bianchi A. Identification of new microRNAs targeting genes regulating the $\mathrm{Pi} / \mathrm{PPi}$ balance in chondrocytes. Biomed Mater Eng. 2014;24:3-16.

31 Kempinska-Podhorodecka A, Milkiewicz M, Wasik U, Ligocka J, Zawadzki M, Krawczyk $\mathrm{M}$, et al. Decreased expression of vitamin D receptor affects an immune response in primary biliary cholangitis via the VDR-miRNA155-SOCS1 pathway. Int J Mol Sci. 2017; 18:289.

32 Mononen N, Lyytikäinen LP, Seppälä I, Mishra PP, Juonala M, Waldenberger M, et al. Whole blood microRNA levels associate with glycemic status and correlate with target mRNAs in pathways important to type 2 diabetes. Sci Rep. 2019;9:1-14.

33 Liu AM, Xu Z, Shek FH, Wong KF, Lee NP, Poon RT, et al. miR-122 targets pyruvate ki- nase M2 and affects metabolism of hepatocellular carcinoma. PloS One. 2014;9:e86872.

34 Zhu Z, Yin J, Li DC, Mao ZQ. Role of microRNAs in the treatment of type 2 diabetes mellitus with Roux-en-Y gastric bypass. Braz J Med Biol Res. 2017;50:e5817.

35 Lu H, Buchan RJ, Cook SA. MicroRNA-223 regulates Glut4 expression and cardiomyocyte glucose metabolism. Cardiovasc Res. 2010;86:410-20.

36 Li Y, Deng S, Peng J, Wang X, Essandoh K, $\mathrm{Mu}$ X, et al. MicroRNA-223 is essential for maintaining functional $\beta$-cell mass during diabetes through inhibiting both FOXO1 and SOX6 pathways. J Biol Chem. 2019.

37 Wander PL, Enquobahrie DA, Bammler TK, Srinouanprachanh S, MacDonald J, Kahn SE, et al. Circulating microRNAs are associated with incident diabetes over 10 years in Japanese Americans. Sci Rep. 2020;10:1-6.

38 Tsukita S, Yamada T, Takahashi K, Munakata Y, Hosaka S, Takahashi H, et al. MicroRNAs $106 \mathrm{~b}$ and 222 improve hyperglycemia in a mouse model of insulin-deficient diabetes via pancreatic $\beta$-cell proliferation. EBioMedicine. 2017;15:163-72.

39 Yang J, Chen Y, Jiang K, Yang Y, Zhao G, Guo $S$, et al. MicroRNA-106a provides negative feedback regulation in lipopolysaccharide-induced inflammation by targeting TLR4. Int J Biol Sci. 2019;15:2308.

40 Kendziorski C, Irizarry RA, Chen KS, Haag JD, Gould MN. On the utility of pooling biological samples in microarray experiments. Proc Natl Acad Sci U S A. 2005;102:4252-7.

41 Guo J, Givens DI, Astrup A, Bakker SJ, Goossens GH, Kratz M, et al. The impact of dairy products in the development of type 2 diabetes: where does the evidence stand in 2019? Adv Nutr. 2019;10:1066-75. 